\title{
Robust fault estimation using an LPV reference model: ADDSAFE benchmark case study
}

\author{
Lejun Chen ${ }^{\mathrm{a}, *}$, Ron Patton ${ }^{\mathrm{b}}$, Philippe Goupil ${ }^{\mathrm{c}}$ \\ ${ }^{a}$ College of Engineering, Mathematics and Physical Sciences, University of Exeter, \\ Exeter, UK, EX4 4QF \\ ${ }^{b}$ School of Engineering, University of Hull, Hull, UK, HU6 7RX \\ ${ }^{c}$ Flight Control System, Airbus Operations S.A.S. 316, Route de Bayonne 31060 \\ Toulouse Cedex 09
}

\begin{abstract}
This paper investigates a mixed $\mathscr{H}_{-} / \mathscr{H}_{\infty}$ linear parameter varying (LPV) fault estimator using an LPV reference estimator. LMIs are used to calculate the affinely parameter-dependent gains of the LPV fault estimator. The design strategy is applied to a high fidelity nonlinear aircraft model provided by AIRBUS for use within the EU-FP7 project ADDSAFE, to estimate the yaw rate sensor faults in the Air Data Inertial Reference System in the presence of the parametric uncertainties. The fault detection performances in various flight conditions are evaluated using the parametric simulation.
\end{abstract}

Keywords: Fault detection and diagnosis, Mixed $\mathscr{H}_{-} / \mathscr{H}_{\infty}$ optimisation, Reference model

\section{Introduction}

\subsection{Background and Motivation}

Model-based fault detection and diagnosis (FDD) has already became a mature subject and has developed from well structured theory in the academic control community with a large amount of work described in (Patton, Frank \& Clark, 2000; Isermann, 1997; Gertler, 1998; Chen \& Patton, 1999;

\footnotetext{
*Corresponding author. Tel: +44-1392-723657

Email addresses: lejun.chen@exeter.ac.uk (Lejun Chen ), r.j.patton@hull.ac.uk (Ron Patton), philippe.goupil@airbus.com (Philippe Goupil)
} 
Isermann, 2005; Ding, 2008; Bokor \& Szabo, 2009). Aerospace applicationbased FDD studies have also been well summarized, e.g. see the book chapter 'Fault detection and diagnosis for aeronautic and aerospace missions' by Henry, Simani and Patton (Edwards, Lombaerts \& Smaili, 2010). An approach to model-based FDD has already been implemented in the AIRBUS industry practice for detecting a Electronic Flight Control Systems failure known as oscillatory failure case (OFC), which can cause a significant increase in the structural load due to erroneous oscillation. When coupled with the flexible modes of the structure, OFC can generate resonance phenomenon and cause unacceptably hight vibration and loads (Goupil, 2010b). Nevertheless, industrial applications of model-based FDD theory for aerospace systems are very limited or restricted (Zolghadri, 2012). The most recent study on the potential of FDD to aircraft flight control industry is the EU-FP7 project ADDSAFE (Advanced Fault Diagnosis for Sustainable Flight Guidance and Control). The aim of the project is to highlight the link between aircraft sustainability and fault detection, it can be demonstrated that improving the fault diagnosis performance in flight control systems facilitates the optimization of the aircraft structural design (resulting in weight saving), which in turn helps to improve aircraft performance and to decrease its environmental footprint (e.g. fuel consumption and noise) (Goupil \& Marcos, 2011, 2012).

The motivation of this paper is to develop and apply a robust fault estimation scheme to estimate and detect the ADDSAFE actuator/sensor faults in above fault scenarios at an early stage of each fault development, in the presence of parametric uncertainties. Especially, the estimator is developed in an affine LPV manner. In recent years, LPV based FDD has been widely developed in the literature (Bokor \& Balas, 2004; Henry, 2008). The most obvious benefit is that the analysis of the performance and stability, together with the synthesis method are established over a wide range of changing parameters. Besides, the LPV design scheme can be considered as an extension of the LTI design scheme. For example, based upon the vertex property (Apkarian et al., 1995), the LPV solution can be calculated by combining multiple LTI solutions calculated on the vertices of the polytope. This property fits well in aerospace gain scheduling designs. Additionally, a trade-off between computational load and design performance can be established by defining a number of suitable scheduling parameters (Marcos \& Balas (2004)). This trade-off is an important part of the industry assessment of the design.

However, from a practical view point, robustness issues associated with plant-model mismatch, aerodynamic database uncertainties, sensor noise and 
imperfect measurements of the scheduling parameters have to be taken into account. The desired performance of the LPV-based design is thus unattainable and the miss detection and false alarm will be generated.

In this paper, above uncertainties are considered parametrically bounded and an LPV reference model based design scheme is derived, based upon using an $\mathscr{H}_{-} / \mathscr{H}_{\infty}$ optimisation technique, to deal with these practical concerns. The purpose of combining $\mathscr{H}_{-}$(Hou \& Patton, 1996) with $\mathscr{H}_{\infty}$ is to allow a trade-off to be established, between sensitivity and robustness of the residual against the fault and disturbance, respectively. In the literature, $\mathscr{H}_{-} / \mathscr{H}_{\infty}$ based approaches can be divided into two categories. One category combines the $\mathscr{H}_{-}$index and $\mathscr{H}_{\infty}$ performance as a multi-objective criterion (Ding et al., 2000; Wang et al., 2007). In another category, the mixed $\mathscr{H}_{-} / \mathscr{H}_{\infty}$ is transformed into a uniform $\mathscr{H}_{\infty}$ problem (Henry \& Zolghadri, 2005), which was extended to an LPV framework by (Grenaille et al., 2008; Henry, 2012). Recent work by Li et al. (2012) proposed a specific $\mathscr{H}_{-}$ index which allows the fault estimation to be achieved in the presence of external disturbances. The parameterizable solution of the fault estimator is then used to construct an $\mathscr{H}_{\infty}$ optimisation procedure.

The idea of using a reference model based design scheme for FDD can be found in Zhong et al. (2003); Frisk \& Nielsen (2006). In design scheme, the reference fault estimator is developed first in the absence of the modeling uncertainty, and the robust fault estimator is then developed to minimize the distance between the reference and robust designs, based upon using the solutions of the reference estimator gains. The parametric uncertainties is thus restrained. Also, non-unique reference designs allow the robustness and stability properties of the robust design to be tuned. Compared with the other state-of-art approaches, this scheme is practical from an engineering point of view as the reference and robust design steps satisfy the requirements of different industrial evaluation phases. In this scheme, a fault estimator with good estimation performance is selected as the reference design evaluated in a preliminary phase of the ADDSAFE project. Also, reference estimator will not be implemented in advanced design phase, which does not generate the extra computational load.

\subsection{Main contribution}

This paper extends the LTI based $\mathscr{H}_{-} / \mathscr{H}_{\infty}$ technique (Li et al., 2012) to LPV system, therefore ensuring wide coverage of the operation conditions. 
On the other hand, an LPV reference model based design scheme is first introduced to mitigate the degradation of the LPV fault estimation performance in the presence of the parametric uncertainties, which includes the imperfect measurements of the LPV system scheduling parameters and aerodynamic uncertainties. This paper applies the proposed scheme to ADDSAFE benchmark problems and demonstrates the robustness of the design via parametric simulation. The fault estimation and detection results have already been evaluated on an industrial benchmark system.

\subsection{Outline of the paper}

The remainder of the paper is outlined as follows: Section 2 introduces some preliminaries associated with ADDSAFE benchmark problem. The fault estimation problem formulation and the technical development are discussed in Section 3. Section 4 describes the design scheme of the proposed fault estimation approach. The ADDSAFE model and the parametric simulation results based upon various fault scenarios of a high-fidelity nonlinear aircraft benchmark model are given in Section 5 .

\subsection{Notation}

The notation and definitions used in the paper are summarized here. For a matrix $A$ with a compatible dimensions, $A^{\prime}, A^{-1}$ and $A^{\dagger}$ denote its transpose, inverse and pseudo-inverse respectively. $A>0(A \geq 0)$ denotes that $A$ is positive (semi-positive) definite. $\operatorname{He}\{A\}$ denotes a shorthand notation for $A+A^{\prime} .\|v\|_{2}$ denotes the frequency domain 2-norm of the signal $v \cdot \mathcal{L}_{2, \Omega}$ is the Lebesgue 2-space, wherein the signal is square integrable and norm bounded in a given finite frequency domain $\Omega$, given by

$$
\mathcal{L}_{2, \Omega}=\left\{v:\|v\|_{2, \Omega}<\infty\right\}
$$

where $\|v\|_{2, \Omega}^{2}=\frac{1}{2 \pi} \int_{\Omega} v^{\prime}(-j \omega) v(j \omega) d \omega$. The Lebesgue 2-space becomes infinitehorizon when $\Omega=[-\infty, \infty]$. Let a system to be denoted in boldface upper case, for example, a parameter dependent system $\mathbf{G}(\rho): u \mapsto y$ is given by

$$
\begin{aligned}
& \dot{x}=A(\rho) x+B(\rho) u \\
& y=C(\rho) x+D(\rho) u
\end{aligned}
$$

where $x, u$, and $y$ denotes the system states, inputs and outputs, respectively. $A(\rho), B(\rho), C(\rho)$, and $D(\rho)$ are affinely parameter-dependent matrices with 
compatible dimensions. $\rho=\left[\rho_{0}, \rho_{1}, \ldots, \rho_{n_{\rho}}\right]^{\prime} \in \Theta \subset \mathscr{R}^{n_{\rho}}$ are the available time-varying scheduling parameters, where $\Theta$ is a compact polytope.

The frequency-domain $\mathscr{H}_{\infty}$ performance and $\mathscr{H}_{-}$index appropriate to a given finite frequency range $\Omega$, are defined based upon the LPV system $\mathbf{G}(\rho)$, as follows

$$
\begin{aligned}
\|\mathbf{G}(\rho)\|_{\infty, \Omega} & =\sup _{\forall \rho \in \Theta, \forall u \in \mathcal{L}_{2, \Omega}} \frac{\|\mathbf{G}(\rho) u\|_{2, \Omega}}{\|u\|_{2, \Omega}}, u \neq 0 \\
\|\mathbf{G}(\rho)\|_{-, \Omega} & =\inf _{\forall \rho \in \Theta, \forall u \in \mathcal{L}_{2, \Omega}} \frac{\|\mathbf{G}(\rho) u\|_{2, \Omega}}{\|u\|_{2, \Omega}}, u \neq 0
\end{aligned}
$$

Remark 1.1. In the literature (see Hou \& Patton (1996); Ding et al. (2000); Jaimoukha et al. (2006); Liu et al. (2005); Henry 85 Zolghadri (2005); Wang et al. (2007)), the $\mathscr{H}_{\infty}$ performance and $\mathscr{H}_{-}$index have been defined via using

a singular value property to measure the sensitivity of the residual against faults corresponding to a given LTI system. The work in Wei $\&$ Verhaegen (2011) is extended in the study of Wang et al. (2007) to one compatible with parameter-varying systems. The $\mathscr{H}_{-}$index over a finite frequency range is also denoted by $\|\cdot\|_{e}$ (Ding et al., 2000; Henry, 2012).

\section{ADDSAFE benchmark}

\subsection{Benchmark problem}

The ADDSAFE benchmark is highly representative of a generic twin engine civil commercial aircraft including the nonlinear rigid-body aircraft model with a full set of control surfaces, actuator models, sensor models, flight control laws and pilot inputs (Goupil \& Puyou, 2011). The model is highly representative of the aircraft flight physics and handling qualities. Three fault scenarios are established within benchmark (Goupil, 2010a).

The Air Data and Inertial Reference System (ADIRS) Monitoring fault scenario defined within the ADDSAFE benchmark is selected as case studies in this paper. Figure.1 shows that the ADIRS in the benchmark contains triplex dedicated sensor redundancy, i.e. three Air Data and Inertial Reference Units (ADIRUs), to measure the states of the aircraft, such as anemometric and inertial data (e.g. angle of attack, load factor and yaw rate). A consolidation process monitors these three redundant measurements and by majority voting chooses the most reliable one as the input of the FCC. 


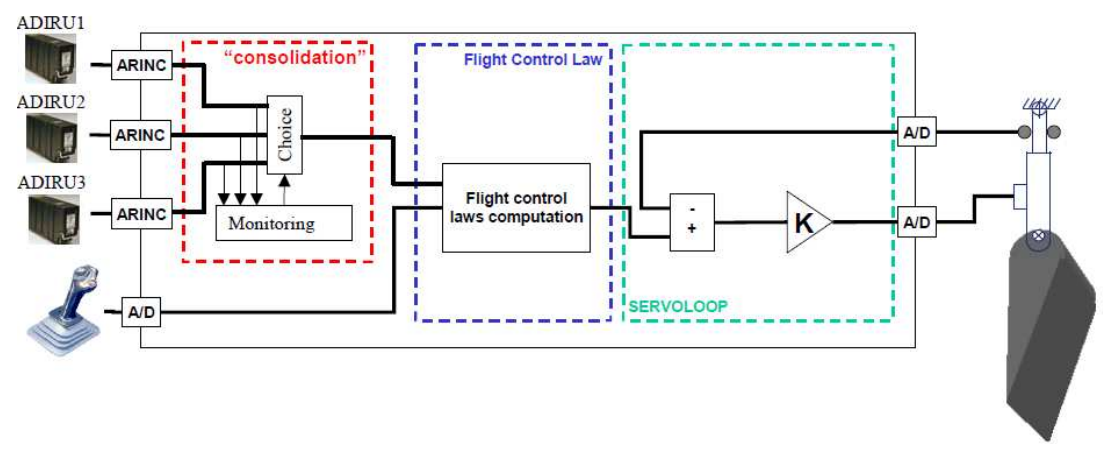

Figure 1: ADIRS monitoring localization in the flight control computer (Goupil, 2010a)

Without successful consolidation, faults occurring on the ADIRUs cause erroneous commands to be sent to the FCC, thereby causing abnormal aircraft behaviour.

Two ADIRS failure cases are defined in scenario, associated with the yaw rate measurement. These are: Case A: Single sensor fails due to the malfunction of an electronic component. Case B: Two sensors fail simultaneously. For Case B, the current industrial practice for fault detection (consolidation process) becomes unreliable, which requires at least two data channels to be fault-free. The robust fault estimation approach proposed in this paper detects and isolates failures for both cases in the presence of parametric uncertainty so that the reliable sensor measurement can be selected as the input of the flight control computer. Several fault types are tested, such as e.g. oscillations, jamming, Non-Return to Zero (NRZ) and noise signals occurring in both case A and case B. Drift and runaway faults occur in case B. All sensor faults in ADDSAFE are modeled in an additive manner, i.e. $y=y_{0}+f$, where $y$ and $y_{0}$ denote the faulty and fault-free sensor measurements. $f$ is the additive sensor fault to be estimated.

\subsection{ADDSAFE LPV model}

The ADDSAFE benchmark is highly representative of a generic twin engine civil commercial aircraft including the nonlinear rigid-body aircraft model with a full set of control surfaces, actuator models, sensor models, flight control laws and pilot inputs (Goupil \& Puyou, 2011).

The affine state-space LPV aircraft rigid body model is established by Hecker (2010), based on using a multivariate polynomial fitting (Pfifer \& 
Hecker, 2011). To build the ADDSAFE LPV model, a set of linear timeinvariant (LTI) state-space systems linearized at certain trim points are obtained at first. Then a least-squares multivariable polynomial fitting is used to interpolate the multiple LTIs to find an LPV model. Since the ADDSAFE fault scenarios are defined only in cruise condition, the LPV modelling using polynomial fitting should not generate large plant-model mismatch. In ADDAFE, the affine LPV model is provided, in particular:

$$
\begin{aligned}
& A(\rho)=A_{0}+A_{1} \rho_{1}+\ldots+A_{n_{\rho}} \rho_{n_{\rho}} \\
& B(\rho)=B_{0}+A_{1} \rho_{1}+\ldots+B_{n_{\rho}} \rho_{n_{\rho}} \\
& C(\rho)=C_{0}+A_{1} \rho_{1}+\ldots+C_{n_{\rho}} \rho_{n_{\rho}} \\
& D(\rho)=D_{0}+A_{1} \rho_{1}+\ldots+D_{n_{\rho}} \rho_{n_{\rho}}
\end{aligned}
$$

The selected scheduling parameters are

$$
\rho=\left[m(k g), X_{c g}(\%), V_{c}(k t), h(f t)\right]
$$

where $m$ is the weight of the aircraft, $X_{c g}$ is the x-axis center gravity, $V_{c}$ denotes the calibrated airspeed and $h$ is the altitude. Instead of using $h$ and $V_{c}$, one may also choose another set of parameters such as true airspeed, Mach number, dynamic pressure to describe the flight envelope. However, the choice of the scheduling parameters was fixed during ADDSAFE project. Since these scheduling parameter are independent from each other, the effective affine LPV region is hyper-rectangular and the scheduling parameters satisfies the polytopic property and vary inside a convex polytope. Suppose $\Theta$ is a compact polytope of vertices $\nu_{1}, \nu_{2}, \ldots, \nu_{r}$, that is

$$
\rho \in \Theta:=\operatorname{Co}\left\{\nu_{1}, \nu_{2}, \ldots, \nu_{r}, r=2^{n_{\rho}}\right\}
$$

where $n_{\rho}=4$ in ADDSAFE. Using vertex property (Apkarian et al., 1995),

$$
A(\rho)=\sum_{i=1}^{r} \alpha_{i} A\left(\nu_{i}\right), \quad \sum_{i=1}^{r} \alpha_{i}=1, \quad \rho=\sum_{i=1}^{r} \alpha_{i} v_{i}, \quad \alpha_{i} \geq 0
$$

\subsection{Evaluation progress}

The performance robustness of the proposed FDD designs are evaluated using the Functional Engineering Simulator (FES). FES is a term used in Space Systems Engineering to describe a software simulator describing the components of a system (including its operating environment) at a functional 
level. FES systems are used in support of the specification, design, verification and operations of space systems, and can be used across the spacecraft development life-cycle, including activities such as system design validation, software verification and validation, spacecraft unit and sub-system test activities (Fernandez et al., 2010). The FES developed by Deimos Space S.L.U. for the ADDSAFE project (Fernandez \& Ramon, 2011) is a non-real-time simulator based on Simulink, Matlab and XML that includes the Airbus benchmark model as well as the robustness and performances metrics for all the fault scenarios defined in the project (Goupil \& Marcos, 2012).

The evaluation process requires repeated closed-loop parametric simulations with various uncertainties and flight conditions to demonstrate robust stability over the whole flight envelope considered. The parametric simulation is thus used to provide an initial robustness verification based on multiple fixed shots inside the flight envelope, listed in Table 1. The units of the shots are widely adopted in aviation use, which are already defined in the ADDSAFE FES. A single shot corresponds to the result of simulation runs with a selected uncertain parameter set.

Table 1: Multiple shots inside flight envelope chosen for parametric simulation

\begin{tabular}{ccccc}
\hline Parameter & \multicolumn{4}{c}{ Shots inside envelope } \\
\hline Altitude $\left(\mathrm{ft} \times 10^{3}\right)$ & 8 & 18 & 28 & 38 \\
Calibrated airspeed (kts) & 160 & 220 & 300 & \\
Mass (ton) & 120 & 180 & 233 & \\
Center of gravity $(\%)$ & 17 & 30 & 41 & \\
\hline
\end{tabular}

For each of the simulation shots in the flight envelope, error bounds on the uncertain aerodynamic parameters, sensor measurements and estimation of physical parameters are applied, according to the information given in Table 2.

Table 2: Aerodynamic database uncertainty bounds, sensor measurements and estimation

\begin{tabular}{ccccc}
\hline Parameter & Variable & Min & Max \\
\hline $\begin{array}{c}\text { Aerodynamic } \\
\text { coefficients }\end{array}$ & $\delta C_{x}, \delta C_{y}, \delta C_{z}$ & $-5 \%$ & 0 & $5 \%$ \\
\hline Measurements & $\delta C_{m}, \delta C_{n}$ & & & \\
\hline Estimation & $\delta m, \delta X_{c g}$ & $-10 \%$ & 0 & $10 \%$ \\
\hline
\end{tabular}


After combining four altitude values, three various values of calibrated airspeeds, mass and center of gravity, and the upper and lower uncertainty bounds of uncertainty listed in Table 1 and Table 2, for each fault, a parametric simulation will cover 324 grid points.

The parametric simulation results are then evaluated based upon the predefined evaluation metrics, including detection time performance (DTP), false alarm rate (FA), missed detection rate (MD) and computational load (ET), etc.

A zero false alarm rate has to be guaranteed in the fault-free situation. Here, six flight conditions or manoeuvres are chosen to be simulated as faultfree. These are: Nose up (abrupt longitudinal manoeuvre), Angle of attack (AOA) protection (triggering of angle of attack protection), Pitch protection (triggering of pitch protection), Yaw angle mode (corresponding to an enhanced auto-pilot hold mode), Turn coordination (coordinated turn) and Cruise phase. For each flight manoeuver, 158 runs are executed for the parametric simulations.

\section{Problem formulation and technical solution}

Consider an affine LPV system subject to actuator and sensor faults and parametric uncertainties, given by

$$
\begin{aligned}
\dot{x} & =(A(\rho)+\delta A(\rho)) x+(B(\rho)+\delta B(\rho)) u+B_{f}(\rho) f+B_{d}(\rho) d \\
y & =(C+\delta C) x+(D+\delta D) u+D_{f} f+D_{d} d
\end{aligned}
$$

where $A(\rho) \in \mathscr{R}^{n \times n}, \delta A(\rho) \in \mathscr{R}^{n \times n}, \delta B(\rho) \in \mathscr{R}^{n \times m}, B(\rho) \in \mathscr{R}^{n \times m}, B_{f}(\rho) \in$ $\mathscr{R}^{n \times q}, B_{d}(\rho) \in \mathscr{R}^{n \times k}, C \in \mathscr{R}^{p \times n}, \delta C \in \mathscr{R}^{p \times n}, D \in \mathscr{R}^{p \times n}, \delta D \in \mathscr{R}^{p \times n}$, $D_{f} \in \mathscr{R}^{p \times q}, D_{d} \in \mathscr{R}^{p \times k}, x \in \mathscr{R}^{n}, u \in \mathscr{R}^{m}$, and $y \in \mathscr{R}^{p}$ represent the system states, inputs and outputs respectively. $f \in \mathscr{R}^{q} \subset \mathcal{L}_{2, \Omega}$ is the vector of sensor or actuator faults. $d \in \mathscr{R}^{k} \subset \mathcal{L}_{2, \Omega}$ represents the external disturbance. Assume the parametric uncertainties can be written as

$$
\left[\begin{array}{cc}
\delta A(\rho) & \delta B(\rho) \\
\delta C & \delta D
\end{array}\right]=\left[\begin{array}{l}
F_{1} \\
F_{2}
\end{array}\right] \Delta\left[\begin{array}{ll}
E_{1} & E_{2}
\end{array}\right]
$$

where $F_{1} \in \mathscr{R}^{n \times n}, F_{2} \in \mathscr{R}^{p \times n}, E_{1} \in \mathscr{R}^{n \times n}$ and $E_{2} \in \mathscr{R}^{n \times m} . \Delta \in \mathscr{R}^{n \times n}$ represents the uncertainty matrix which satisfies $\Delta \Delta^{\prime} \leq I$.

Assumption 3.1. (i) $(C, A(\rho))$ is detectable, $\forall \rho \in \Theta$.(ii) Matrix $D_{f}$ is full column rank. (iv) $\mathbf{G}_{f}(\rho): f \mapsto y$ has no zeros on the extended imaginary axis, $\forall \rho \in \Theta$. 
Remark 3.1. In Assumption 3.1, (i) guarantees that, $\forall \rho \in \Theta$, there exists $L(\rho)$ such that $A(\rho)+L(\rho) C$ is negative, which can be verified on all vertices of the polytope. (ii) is necessary for the existence of the solution of the LPV fault estimator, which is relaxed by Wang \& Yang (2008). (iv) is necessity of the existence of a stable fault estimator.

Consider an faulty LPV system without external disturbances or parametric uncertainties, i.e. $d=0$, and $\Delta=0$. The reference fault estimator is in the form of

$$
\begin{aligned}
\dot{\hat{x}}_{f} & =A(\rho) \hat{x}_{f}+B(\rho) u-L_{1}(\rho)\left(y_{f}-\hat{y}_{f}\right) \\
\hat{y}_{f} & =C \hat{x}_{f}+D u \\
r_{f} & =L_{2}\left(y_{f}-\hat{y}_{f}\right)
\end{aligned}
$$

where $L_{1}(\rho) \in \mathscr{R}^{n \times p}$ and $L_{2} \in \mathscr{R}^{q \times p}$ are reference estimator gains. $y_{f} \in \mathscr{R}^{p}$ is the reference system output and $r_{f} \in \mathscr{R}^{q}$ is the reference fault estimate. Let $e_{f}=x-\hat{x}_{f} \in \mathscr{R}^{n}$, it is shown that,

$$
\begin{aligned}
& \dot{e}_{f}=\underbrace{\left(A(\rho)+L_{1}(\rho) C\right)}_{\bar{A}(\rho)} e_{f}+\underbrace{\left(B_{f}(\rho)+L_{1}(\rho) D_{f}\right)}_{\bar{B}(\rho)} f \\
& r_{f}=\underbrace{L_{2} C}_{\bar{C}} e_{f}+\underbrace{L_{2} D_{f}}_{\bar{D}} f
\end{aligned}
$$

Define $\mathbf{G}_{r_{f} f}(\rho): r_{f} \mapsto f$, an ideal fault estimator requires that $\mathbf{G}_{r_{f} f}(\rho)=\mathbf{I}$ which is equivalent to finding a fault estimator to achieve a specific $\mathscr{H}_{-}$index $\left\|\mathbf{G}_{r_{f} f}(\rho)\right\|_{-} \geq 1$ since $\|\mathbf{I}\|_{-} \geq 1$. Since $L_{2} \neq 0$, the necessary and sufficient condition to achieve $\mathbf{G}_{r_{f} f}(\rho)=\mathbf{I}$ is $\bar{B}(\rho)=0$ and $\bar{D}=I, \forall \rho \in \Theta$. It follows from the work in (Li et al., 2012) that the corresponding solutions of $L_{1}(\rho)$ and $L_{2}$ are given by

$$
\begin{gathered}
L_{1}(\rho)=-M(\rho)+Z_{1} D_{f}^{\perp} \\
L_{2}=D_{f}^{\dagger}+Z_{2} D_{f}^{\perp}
\end{gathered}
$$

where $Z_{1} \in \mathscr{R}^{n \times q}$ and $Z_{2} \in \mathscr{R}^{q \times q}$ are free matrices. $M(\rho) \in \mathscr{R}^{n \times p}$ and $D_{f}^{\perp} D f=0, M(\rho)=B_{f}(\rho) D_{f}^{\dagger}$. The methodology of finding $Z_{1}$ and $Z_{2}$ for the LTI system is proposed in (Li et al., 2012), which can be easier extended into an LPV framework using vertex property. The details are ignored here due to the length of the paper. 
In the following, the parametric uncertainties are considered. The robust fault estimator is defined as

$$
\mathbf{F}(\rho):\left\{\begin{array}{l}
\dot{\hat{x}}_{a}=A(\rho) \hat{x}_{a}+B(\rho) u-L_{1}^{*}(\rho)\left(y_{a}-\hat{y}_{a}\right) \\
\hat{y}_{a}=C \hat{x}_{a}+D u \\
r_{a}=L_{2}^{*}\left(y_{a}-\hat{y}_{a}\right)
\end{array}\right.
$$

where $L_{1}^{*}(\rho) \in \mathscr{R}^{n \times p}$ and $L_{2}^{*} \in \mathscr{R}^{q \times p}$ are robust estimator gains. $r_{a} \in \mathscr{R}^{q}$ is the robust fault estimate. $y_{a} \in \mathscr{R}^{p}$ is uncertain system output. Let $e_{a}=x-\hat{x}_{a} \in \mathscr{R}^{n}$, it follows

$$
\begin{aligned}
& \dot{e}_{a}=\underbrace{\left(A(\rho)+L_{1}^{*}(\rho) C\right)}_{\bar{A}_{a}(\rho)} e_{a}+(\underbrace{\delta A(\rho)+L_{1}^{*}(\rho) \delta C}_{\delta \bar{A}_{a x}(\rho)}) x \\
& +\underbrace{\left(\delta B(\rho)+L_{1}^{*}(\rho) \delta D\right.}_{\delta \bar{B}_{a u}(\rho)}) u+\underbrace{\left(B_{f}(\rho)+L_{1}^{*}(\rho) D_{f}\right)}_{\bar{B}_{a f}(\rho)} f \\
& +\underbrace{\left(B_{d}(\rho)+L_{1}^{*}(\rho) D_{d}\right)}_{\bar{B}_{a d}(\rho)} d \\
& r_{a}=\underbrace{L_{2}^{*} C}_{\bar{C}_{a}} e_{a}+\underbrace{L_{2}^{*} \delta C}_{\delta \bar{C}_{a x}} x+\underbrace{L_{2}^{*} \delta D}_{\delta \bar{D}_{a u}} u+\underbrace{L_{2}^{*} D_{f}}_{\bar{D}_{a f}} f+\underbrace{L_{2}^{*} D_{d}}_{\bar{D}_{a d}} d
\end{aligned}
$$

The robust fault estimator can also be written as

$$
r_{a}=\mathbf{G}_{r_{a} x}(\rho) x+\mathbf{G}_{r_{a} u}(\rho) u+\mathbf{G}_{r_{a} f}(\rho) f+\mathbf{G}_{r_{a} d}(\rho) d
$$

where $\mathbf{G}_{r_{a} x}(\rho): x \mapsto r_{a}, \mathbf{G}_{r_{a} u}(\rho): u \mapsto r_{a}, \mathbf{G}_{r_{a} f}(\rho): f \mapsto r_{a}$, and $\mathbf{G}_{r_{a} d}(\rho):$ $d \mapsto r_{a}$. Note that the robust fault estimate depends not only on faults, but also on the inputs and states. Therefore, the design of a robust fault estimator to mitigate the performance degradation caused by parametric uncertainties is the main design objective of this work. The robust fault estimation problem can now be formulated as an $\mathscr{H}_{\infty}$ model mismatching problem:

Problem 3.1. Given a system in Eq.(9), find a stable robust fault estimator with gain matrices $L_{1}^{*}(\rho)$ and $L_{2}^{*}$ to generate a fault estimate $r_{a}$, achieving the following infimum for Eq.(17):

$$
\gamma:=\inf _{\forall \rho \in \Theta}\left\{\left\|\mathbf{G}_{r_{e} w}(\rho)\right\|_{\infty, \Omega}:\left\|\mathbf{G}_{r_{a} f}(\rho)\right\|_{-, \Omega} \geq 1\right\}
$$




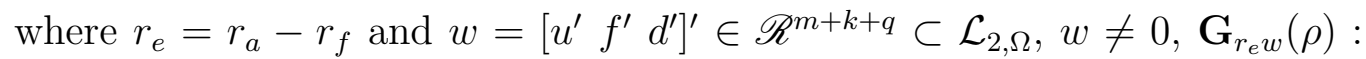
$w \mapsto r_{e}$. Recall that the dynamics of the robust and reference fault estimators shown in Eq.(17) and Eq.(16) and augment the states to be $\left[e_{a} e_{f} x\right]^{\prime}$, then

$$
\left\|\mathbf{G}_{r_{e} w}(\rho)\right\|_{\infty, \Omega}=\left\|\left[\mathbf{G}_{r_{a} u}(\rho) \mathbf{G}_{r_{a} f}(\rho)-\mathbf{I} \mathbf{G}_{r_{d} d}(\rho)\right]\right\|_{\infty, \Omega}
$$

In Eq.(19), $\mathbf{G}_{r_{a} u}(\rho)=\mathbf{F}(\rho) \mathbf{G}_{u}(\rho), \mathbf{G}_{r_{a} f}(\rho)=\mathbf{F}(\rho) \mathbf{G}_{f}(\rho)$, and $\mathbf{G}_{r_{d} d}(\rho)=$ $\mathbf{F}(\rho) \mathbf{G}_{d}(\rho)$, where $\mathbf{G}_{u}(\rho): u \mapsto y, \mathbf{G}_{f}(\rho): f \mapsto y$, and $\mathbf{G}_{d}(\rho): d \mapsto y$. Based upon Lemma 3.1, $\left\|\mathbf{G}_{r_{a} f}(\rho)\right\|_{-, \Omega} \geq 1$ is equivalent to $\mathbf{G}_{r_{a} f}(\rho)=\mathbf{I}$, which allows the ideal fault estimation to be achieved.

Lemma 3.1. (Extension of the work of Li et al. (2012)) Suppose that there exists stable fault estimators $\tilde{\mathbf{F}}(\rho)$ and $\mathbf{F}(\rho)$ to achieve infimum $\gamma$ and $\gamma^{\prime}$, such that

$$
\begin{gathered}
\gamma:=\inf _{\forall \rho \in \Theta}\left\{\left\|\tilde{\mathbf{G}}_{r_{e} w}(\rho)\right\|_{\infty, \Omega}: \tilde{\mathbf{G}}_{r_{a} f}(\rho)=\boldsymbol{I}\right\} \\
\gamma^{\prime}:=\inf _{\forall \rho \in \Theta}\left\{\left\|\mathbf{G}_{r_{e} w}(\rho)\right\|_{\infty, \Omega}:\left\|\mathbf{G}_{r_{a} f}(\rho)\right\|_{-, \Omega} \geq 1\right\}
\end{gathered}
$$

where $\tilde{\mathbf{G}}$ represents systems which contain estimator $\tilde{\mathbf{F}}(\rho)$. Then, $\gamma=\gamma^{\prime}$.

Proof. As defined in Eq.(11), let $\tilde{\mathbf{F}}(\rho)$ and $\mathbf{F}(\rho)$ be two stable reference fault estimators, satisfying Eq.(20) and Eq.(21), respectively. There always exists an invertible system transformation $\mathbf{X}(\rho)$, such that $\tilde{\mathbf{F}}(\rho)=\mathbf{X}(\rho) \mathbf{F}(\rho)$. Since $\tilde{\mathbf{G}}_{r_{a} f}(\rho)=\tilde{\mathbf{F}}(\rho) \mathbf{G}_{f}(\rho)=\mathbf{I}, \mathbf{X}(\rho) \mathbf{F}(\rho) \mathbf{G}_{f}(\rho)=\mathbf{I}$ and $\mathbf{X}(\rho)=\left(\mathbf{F}(\rho) \mathbf{G}_{f}(\rho)\right)^{-1}$. Note that $\left\|\mathbf{F}(\rho) \mathbf{G}_{f}(\rho)\right\|_{-, \Omega} \geq 1$, then $\left\|\mathbf{X}^{-1}(\rho)\right\|_{-, \Omega} \geq 1$. It is not hard to show that

$$
\|\mathbf{I}\|_{\infty, \Omega} \geq\|\mathbf{X}(\rho)\|_{-, \Omega}\left\|\mathbf{X}^{-1}(\rho)\right\|_{\infty, \Omega}
$$

Therefore, $\|\mathbf{X}(\rho)\|_{\infty, \Omega}<1$. According to Eq. (19),

$$
\begin{aligned}
& \left\|\tilde{\mathbf{G}}_{r_{e} w}(\rho)\right\|_{\infty, \Omega}=\left\|\tilde{\mathbf{F}}(\rho)\left[\mathbf{G}_{u}(\rho) \quad 0 \quad \mathbf{G}_{d}(\rho)\right]\right\|_{\infty, \Omega} \\
& =\left\|\mathbf{X}(\rho) \mathbf{F}(\rho)\left[\mathbf{G}_{u}(\rho) \quad 0 \quad \mathbf{G}_{d}(\rho)\right]\right\|_{\infty, \Omega} \\
& \leq\|\mathbf{X}(\rho)\|_{\infty, \Omega}\left\|\mathbf{F}(\rho)\left[\mathbf{G}_{u}(\rho) \quad 0 \quad \mathbf{G}_{d}(\rho)\right]\right\|_{\infty, \Omega} \\
& \leq\left\|\mathbf{F}(\rho)\left[\begin{array}{lll}
\mathbf{G}_{u}(\rho) & 0 & \mathbf{G}_{d}(\rho)
\end{array}\right]\right\|_{\infty, \Omega}
\end{aligned}
$$

Hence, $\gamma \leq \gamma^{\prime}$. Since $\|\mathbf{I}\|_{-} \geq 1$ implies $\gamma \geq \gamma^{\prime}$, it concludes that $\gamma=\gamma^{\prime}$.

The structure of the fault estimator is unchanged, allowing the design freedom to be further exploited. The solutions of $L_{1}^{*}(\rho)$ and $L_{2}^{*}$ to approximate a fault estimation $\mathbf{G}_{r_{a} f}(\rho)=\mathbf{I}$ are given by

$$
L_{1}^{*}(\rho)=-M(\rho)+Z_{1}^{*} D_{f}^{\perp}
$$




$$
L_{2}^{*}=D_{f}^{\dagger}+Z_{2}^{*} D_{f}^{\perp}
$$

where $Z_{1}^{*} \in \mathscr{R}^{n \times q}$ and $Z_{2}^{*} \in \mathscr{R}^{q \times q}$ are free design matrices.

Suppose the reference design is available, Theorem 3.1 is now proposed to determine the robust solutions.

Theorem 3.1. $\gamma$ defined in Eq.(18) is achieved iff there exists s.p.d matrices $P_{1} \in \mathscr{R}^{n \times n}, P_{2} \in \mathscr{R}^{n \times n}$ and $P_{3} \in \mathscr{R}^{n \times n}$, full matrices $S^{*} \in \mathscr{R}^{n \times q}$ and $Z_{2}^{*}$, such that

$$
N(\rho)_{[8 \times 8]}<0
$$

where $N(\rho)$ is symmetric and $N(\rho)_{[a, b]}$ represents the $(a, b)$ block element of $N(\rho) . Z_{1}$ and $Z_{2}$ are known matrices calculated at the reference estimator design step and $Z_{1}^{*}=P_{1}^{-1} S^{*}$. In (25), for $a \leq b$ :

$$
\begin{aligned}
& N(\rho)_{1,1}=\operatorname{He}\left\{P_{1}(A(\rho)-M(\rho) C)+S^{*} D_{f}^{\perp} C\right\} \\
& N(\rho)_{1,6}=P_{1}\left(B_{d}(\rho)-M(\rho) D_{d}\right)+S^{*} D_{f}^{\perp} D_{d} \\
& N(\rho)_{1,7}=\left(D_{f}^{\dagger} C\right)^{\prime}+\left(Z_{2}^{*} D_{f}^{\perp} C\right)^{\prime} \\
& N(\rho)_{1,8}=P_{1}\left(F_{1}-M(\rho) F_{2}\right)+S^{*} D_{f}^{\perp} F_{2} \\
& N(\rho)_{2,2}=\operatorname{He}\left\{P_{2}\left(A(\rho)-M(\rho) C+Z_{1} D_{f}^{\perp} C\right)\right\} \\
& N(\rho)_{2,7}=-\left(D_{f}^{\dagger} C\right)^{\prime}-\left(Z_{2} D_{f}^{\perp} C\right)^{\prime} \\
& N(\rho)_{3,3}=\operatorname{He}\left\{P_{3} A(\rho)\right\}+E_{1}^{\prime} E_{1}, N(\rho)_{3,4}=P_{3} B(\rho)+E_{1}^{\prime} E_{2} \\
& N(\rho)_{3,5}=P_{3} B_{f}(\rho), N(\rho)_{3,6}=P_{3} B_{d}(\rho), N(\rho)_{3,8}=P_{3} F_{1} \\
& N(\rho)_{4,4}=-\gamma I+E_{2}^{\prime} E_{2}, \quad N(\rho)_{5,5}=-\gamma I \quad N(\rho)_{6,6}=-\gamma I \\
& N(\rho)_{6,7}=D_{d}^{\prime}\left(\left(Z_{2}^{*}-Z_{2}\right) D_{f}^{\perp}\right)^{\prime}, \quad N(\rho)_{7,7}=-\gamma I \\
& N(\rho)_{7,8}=\left(D_{f}^{\dagger}+Z_{2}^{*} D_{f}^{\perp}\right) F_{2}, \\
& N(\rho)_{a, b} \text { are null, for all other indices. }
\end{aligned}
$$

Remark 3.2. Due to the vertex property, the same result can be found by a combination of a limited number of solutions calculated using

$$
\left[N\left(\nu_{i}\right)\right]_{8 \times 8}<0, i=1,2, \ldots, r
$$

where $\nu_{i}$ denotes the set of scheduling parameters on the ith vertex.

Remark 3.3. Li et al. (2012) points out that the resulting gain matrices may not able to stablise the observer. In this case, an inner-outer factorization 
must be performed and the fault estimation thus cannot be achieved. However, by selecting various $Z_{1}$ and $Z_{2}$ for reference model, various robust design gains $L_{1}^{*}(\rho)$ and $L_{2}^{*}$ could be deduced to avoid losing the estimation property. The systematic tuning procedure is not exploited in this paper.

\section{Design scheme}

The design scheme mainly contains two steps:

- Step 1 (ADDSAFE preliminary phase): Reference fault estimator design.

In this step, let $d=0$ and matrices $\delta A(\rho), \delta B(\rho), \delta C$, and $\delta D$ be null. Calculate the matrices $Z_{1}$ and $Z_{2}$ using the vertex property and the method proposed in (Li et al., 2012), and substitute them into (13) and (14) to find out $L_{1}(\rho)$ and $L_{2}$. Implement the reference fault estimator in (11) to generate the reference fault estimation signal $r_{f}$ and then evaluate the fault estimation performance in preliminary phase of the project.

- Step 2 (ADDSAFE advanced phase): Robust fault estimator design.

In Step 2, matrices $\delta A(\rho), \delta B(\rho), \delta C$, and $\delta D$ are assumed not null. Set the reference fault estimator deduced in Step 1 as the reference design, i.e. only save the matrices $Z_{1}$ and $Z_{2}$ calculated in Step 1. Then solving the LMI in (25) to calcuate the matrices $Z_{1}^{*}$ and $Z_{2}^{*}$. Substitute matrices $Z_{1}^{*}$ and $Z_{2}^{*}$ to (23) and (24), respectively, to calculate the LPV observer gains $L_{1}^{*}(\rho)$ and $L_{2}^{*}$. Implement observer (15) to generate the robust fault estimate $r_{a}$ for the isolation and decision making purposes.

The FDD structure of the proposed approach is shown in Fig. 2.

\section{ADDSAFE simulation results}

In ADDSAFE sensor fault scenario, the external disturbances are assumed to be zero. An open-loop LPV state-space aircraft rigid body model $(A(\rho), B(\rho), C(\rho)$ and $D(\rho))$ is obtained through using the multivariable polynominal interpolation as discussed in Section 2.2. Then, Eq.(9) can be written as

$$
\begin{aligned}
& \dot{x}=\left(A(\rho)+F_{1} \Delta E_{1}\right) x+\left(B(\rho)+F_{1} \Delta E_{2}\right) u \\
& y=\left(C+F_{2} \Delta E_{1}\right) x+\left(D+F_{2} \Delta E_{1}\right) u+D_{f} f
\end{aligned}
$$




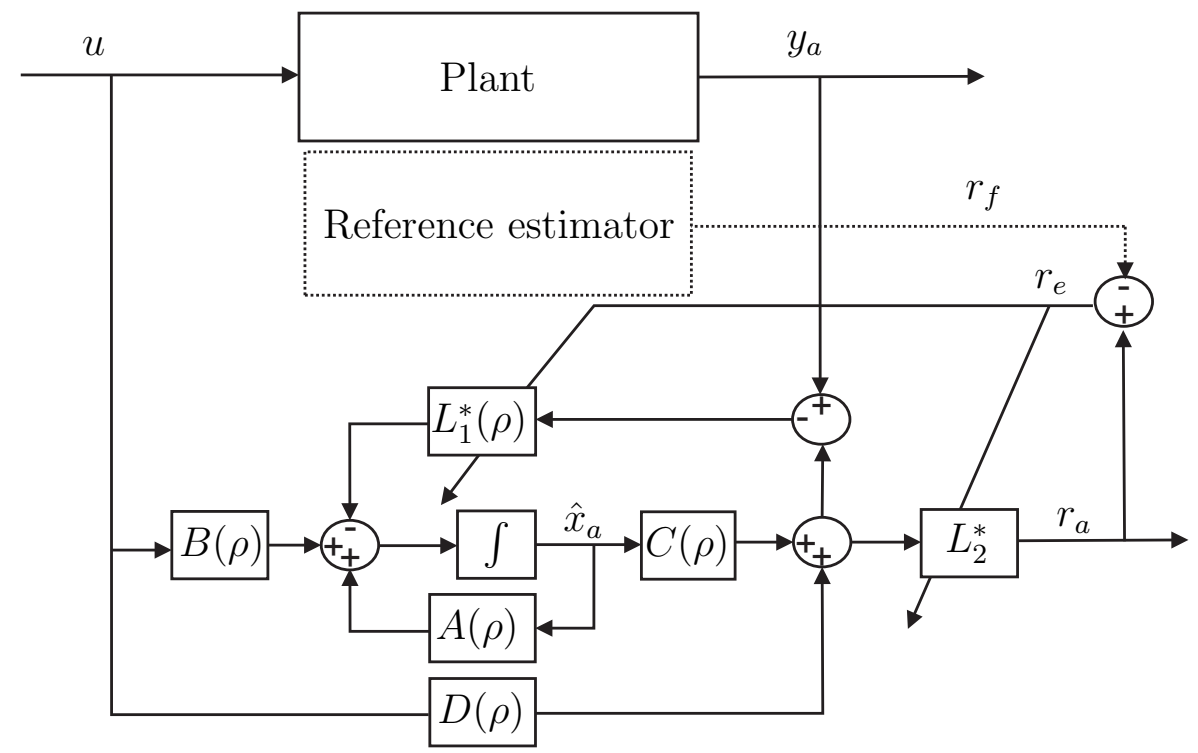

Figure 2: FDD system structure of reference model based design

where matrices $F_{1}, F_{2}, E_{1}, E_{2}$ and $\Delta$ (defined in Eq.(10)) are calculated by Pulecchi \& Marcos (2011), based on using a Linear Fractional Transformationbased realization. $y=\left[\phi, p, r_{1}, r_{2}, r_{3}\right]^{\prime}$ and $x=\left[\phi, v_{y}, p, r\right]^{\prime}$ are the aircraft body axis outputs and states, respectively. $\phi$ is bank angle, $p$ is roll rate, $r_{1}$, $r_{2}$ and $r_{3}$ are yaw rate signals senses on three ADIRUs, wherein $r_{1}$ and $r_{2}$ are two measurements possibly corrupted by the additive sensor fault $f$ in Case A or Case $\mathrm{B}$, and $r_{3}$ is always fault-free. States $v_{y}$ and $r$ represent the aircraft velocity along $y$-axis and the yaw rate signal after the consolidation logic, respectively, which are assumed to be unavailable. $u_{b}$ contains the set of all lateral control surface deflections (i.e. four ailerons, eight spoilers plus one rudder) together with a horizontal wind speed input along the aircraft body axis $u_{w}{ }^{1}$. Note that for the ailerons and spoilers, the control surface sensors are massing, the deflections information are measured via using their rod sensors.

The parametric simulation results are all generated under the cruise manoeuvre with various flight conditions and the parametric uncertainties listed

\footnotetext{
${ }^{1} \mathrm{In}$ ADDSAFE, the wind effect is delivered (i.e. $u_{w}=0$ )
} 
in Table 1 and Table 2. A combination of Table 1 and Table 2 leads to a total 324 simulation runs for each type of the fault. All faults are assumed to be occurred from 2 sec. All fault types within ADDSAFE sensor fault scenario described in Section 2.1 are investigated. In this section, only the fault estimation results and the fault detection metric results are presented due to the large amount of the data generated within the parametric simulation. Note that the numerical results are not included here. This is due to the industrial restricts. As described in Section 2.1, the oscillations, jamming, NRZ and noise occur in both case A and case B. Drift and runaway faults occur in case B. For the oscillation cases A and B, the fault amplitudes are $1.25 \%$ and $3.75 \%$ of the admissible range. For the jamming cases in $\mathrm{A}$ and $\mathrm{B}$, the fault amplitudes are $1.25 \%$ and $5 \%$ of the admissible ranges. The fault amplitude of the NRZ fault is $320 \%$ of the admissible range. Also, for the noise cases $\mathrm{A}$ and $\mathrm{B}$, the covariances of the noise signals are 0.5 and 20 , respectively.

The normalized parametric simulation results are shown in Figs. A.3, A.4 and Fig. A.5. For various fault types and cases, the top figures always show the fault estimation signals associated with the first yaw rate ADIRU fault (added on $r_{1}$ ) and the bottom figures always depict the fault estimation signals associated withe the second yaw rate ADIRU faults (added on $r_{2}$ ). It is clear from Figs. A.3 that, for oscillation fault and jamming fault types, the fault amplitudes and fault occurrence time are well estimated and the case A and the case B can be isolated. Note that the oscillation appears in case B with jamming fault, this is due to the unexpected closed-loop aircraft behaviour affected by the jamming faults. The fault estimation results associated with the drift and runaway fault types are shown in Figs. A.4, the fault occurrence time and the rate of the runaway/drift are correctly estimated. For noise A, noise B, NRZ A and NRZ B fault types, Fig. A.5 shows that the faults are estimated despite various NRZ frequencies and covariances of the noise signals, and case A and case B can be well isolated. Above all simulation results show that the actual FDD estimator is robust so that the parametric uncertainty does not significantly affect the estimation results.

The corresponding evaluation results of the parametric simulation are given in Table 3. Clearly, both the false alarm rate (FA) and miss detection rate (MD) are zero at various flight points despite various parametric uncertainties. For detection time performance (DTP), the value of ' 1 ' indicates that the fault is detected at the required detection time, and any value between ' 0 ' and ' 1 ' indicates a faster detection time. Table 3 shows that the 
detection time performance is small enough for all types of the faults, which implies that the faults can be detected rapidly after their occurrences. The mean and variance of the fault estimation bias, associated with two sensor channels $\left(r_{2}\right.$ and $\left.r_{2}\right)$, are also listed in Table 3 .

\section{Conclusion}

This paper describes the applications of robust FDD the ADIRS yaw rate sensor fault scenario defined in the ADDSAFE project. An $\mathscr{H}_{-} / \mathscr{H}_{\infty}$ LPV fault estimation approach is proposed, based on using a reference design. Simulation results demonstrate the robustness of the fault estimation against the parametric uncertainties caused by aerodynamic database uncertainties and measurement/estimation errors of the physical parameters. The reliability of proposed method is also evaluated via parametric campaign.

\section{Acknowledgments}

The authors acknowledge funding support by the European Commission for the contract FP7-233815, Advanced Fault Diagnosis for Sustainable Flight Guidance and Control (ADDSAFE), led by Dr. Andres Marcos (Deimos Space, Spain).

\section{Appendix A. Proof of Theorem 3.1}

Proof. Define

$$
\begin{aligned}
& \hat{A}(\rho)= {\left[\begin{array}{ccc}
\bar{A}_{a}(\rho) & 0 & \delta \bar{A}_{a x}(\rho) \\
0 & \bar{A}(\rho) & 0 \\
0 & 0 & A(\rho)+\delta A(\rho)
\end{array}\right] } \\
& \hat{B}(\rho)= {\left[\begin{array}{ccc}
\delta \bar{B}_{a u}(\rho) & \bar{B}_{a f}(\rho) & \bar{B}_{a d}(\rho) \\
0 & \bar{B}(\rho) & 0 \\
B(\rho)+\delta B(\rho) & B_{f}(\rho) & B_{d}(\rho)
\end{array}\right] } \\
& \hat{C}=\left[\begin{array}{lll}
\bar{C}_{a} & -\bar{C} & \delta \bar{C}_{a x}
\end{array}\right] \\
& \hat{D}=\left[\begin{array}{lll}
\delta \bar{D}_{a u} & \bar{D}_{a f}-\bar{D} & \bar{D}_{a d}
\end{array}\right]
\end{aligned}
$$


Table 3: Fault estimation performance evaluation results

\begin{tabular}{ccccccc}
\hline \multirow{2}{*}{ Fault type } & \multicolumn{6}{c}{ Evaluation results } \\
\cline { 2 - 7 } & & FA(\%) & MD $(\%)$ & $\begin{array}{c}\text { Min/Mean } \\
\text { /Max DTP }\end{array}$ & $\begin{array}{c}\text { Mean value }\left(r_{1} / r_{2}\right) \\
\text { (Estimation BIAS) }\end{array}$ & $\begin{array}{c}\text { Estimation Variance }\left(r_{1} / r_{2}\right) \\
\text { Variance }(\text { Estimation })\end{array}$ \\
\hline \multirow{2}{*}{ Oscillation } & $\mathrm{A}$ & 0 & 0 & 0.002 & $1.139 \mathrm{e}-5 /-1.795 \mathrm{e}-20$ & $6.899 \mathrm{e}-4 / 7.102 \mathrm{e}-19$ \\
\cline { 2 - 7 } & $\mathrm{B}$ & 0 & 0 & 0.333 & $3.853 \mathrm{e}-5 / 1.140 \mathrm{e}-5$ & $2.331 \mathrm{e}-4 / 6.899 \mathrm{e}-4$ \\
\hline \multirow{2}{*}{ Jamming } & $\mathrm{A}$ & 0 & 0 & 0.002 & $-0.0115 /-1.795 \mathrm{e}-20$ & $0.012 / 7.102 \mathrm{e}-19$ \\
\cline { 2 - 7 } & $\mathrm{B}$ & 0 & 0 & 0.333 & $-0.030 /-0.030$ & $0.070 / 0.070$ \\
\hline \multirow{2}{*}{ Drift } & & 0 & 0 & 0.333 & $0.377 / 0.377$ & $0.100 / 0.100$ \\
\hline \multirow{2}{*}{ Runaway } & & 0 & 0 & 0.233 & $4.032 / 4.032$ & $1.373 / 1.373$ \\
\hline \multirow{2}{*}{ NRZ } & $\mathrm{A}$ & 0 & 0 & 0.002 & $-7.303 \mathrm{e}-4 /-1.381 \mathrm{e}-20$ & $8.629 \mathrm{e}-4 / 7.657 \mathrm{e}-19$ \\
\cline { 2 - 7 } & $\mathrm{B}$ & 0 & 0 & 0.333 & $-0.342 /-0.342$ & $0.675 / 0.675$ \\
\hline \multirow{2}{*}{ Noise } & $\mathrm{A}$ & 0 & 0 & 0.002 & $-3.908 \mathrm{e}-4 /-1.795 \mathrm{e}-20$ & $0.083 / 7.102 \mathrm{e}-19$ \\
\cline { 2 - 7 } & $\mathrm{B}$ & 0 & 0 & 0.333 & $-3.9075 \mathrm{e}-4 /-3.908 \mathrm{e}-4$ & $0.083 / 0.083$ \\
\hline
\end{tabular}


Let $e=\left[\begin{array}{lll}e_{a} & e_{f} & x\end{array}\right]^{\prime}$ and combine Eq.(12), Eq.(13), Eq.(14), Eq.(16), Eq.(23) and Eq.(24) to yield

$$
\begin{aligned}
\dot{e} & =\hat{A}(\rho) e+\hat{B}(\rho) w \\
r_{e} & =\hat{C}(\rho) e+\hat{D}(\rho) w
\end{aligned}
$$

Based on the Bounded Real Lemma (Apkarian et al., 1995), a sufficient condition to ensure stability of Eq.(A.1) and $\mathscr{H}_{-} / \mathscr{H}_{\infty}$ performance $\gamma$ shown in Eq.(18) is given by

$$
\hat{M}_{B R L}=M_{B R L}+\delta M_{B R L}<0
$$

where,

$$
\begin{aligned}
& M_{B R L}=\left[\begin{array}{ccc}
\operatorname{He}\left\{P_{1} \bar{A}_{a}(\rho)\right\} & 0 & 0 \\
* & \operatorname{He}\left\{P_{2} \bar{A}(\rho)\right\} & 0 \\
* & * & \operatorname{He}\left\{P_{3} A(\rho)\right\} \\
* & * & * \\
* & * & * \\
* & * & * \\
* & * & *
\end{array}\right. \\
& 0 \quad P_{1} \bar{B}_{a f}(\rho) \quad P_{1} \bar{B}_{a d}(\rho) \quad \bar{C}_{a}^{\prime} \\
& \begin{array}{llll}
0 & P_{2} \bar{B}(\rho) & 0 & -\bar{C}^{\prime}
\end{array} \\
& P_{3} B(\rho) \quad P_{3} B_{f}(\rho) \quad P_{3} B_{d}(\rho) \quad 0 \\
& \begin{array}{llll}
-\gamma I & 0 & 0 & 0
\end{array} \\
& \text { * } \quad-\gamma I \quad 0 \quad \bar{D}_{a f}^{\prime}-\bar{D}^{\prime} \\
& * \quad *^{*} \quad-\gamma I \quad \bar{D}_{a d}^{\prime} \\
& \begin{array}{llll}
* & * & * & -\gamma I
\end{array}
\end{aligned}
$$


Let

$$
\begin{aligned}
& F_{\tau}=\left[\begin{array}{c}
P_{1} F_{1}+P_{1} L_{1}^{*}(\rho) F_{2} \\
0 \\
P_{3} F_{1} \\
0 \\
0 \\
0 \\
L_{2}^{*} F_{2}
\end{array}\right] \\
& E_{\tau}=\left[\begin{array}{lllllll}
0 & 0 & E_{1} & E_{2} & 0 & 0 & 0
\end{array}\right]
\end{aligned}
$$

Then, $\delta M_{B R L}$ can then be rewritten as

$$
\delta M_{B R L}=F_{\tau} \Delta E_{\tau}+E_{\tau}^{\prime} \Delta^{\prime} F_{\tau}^{\prime}
$$

Since $\Delta \Delta^{\prime} \leq I$, substituting Eq.(A.7) into Eq.(A.2) and using the Schur complement to yield

$$
\left[\begin{array}{cc}
M_{B R L}+E_{\tau}^{\prime} E_{\tau} & F_{\tau} \\
F_{\tau}^{\prime} & -I
\end{array}\right]<0
$$

Substituting Eq.(A.3), Eq.(A.5) and Eq.(A.6) into Eq.(A.8) leads to:

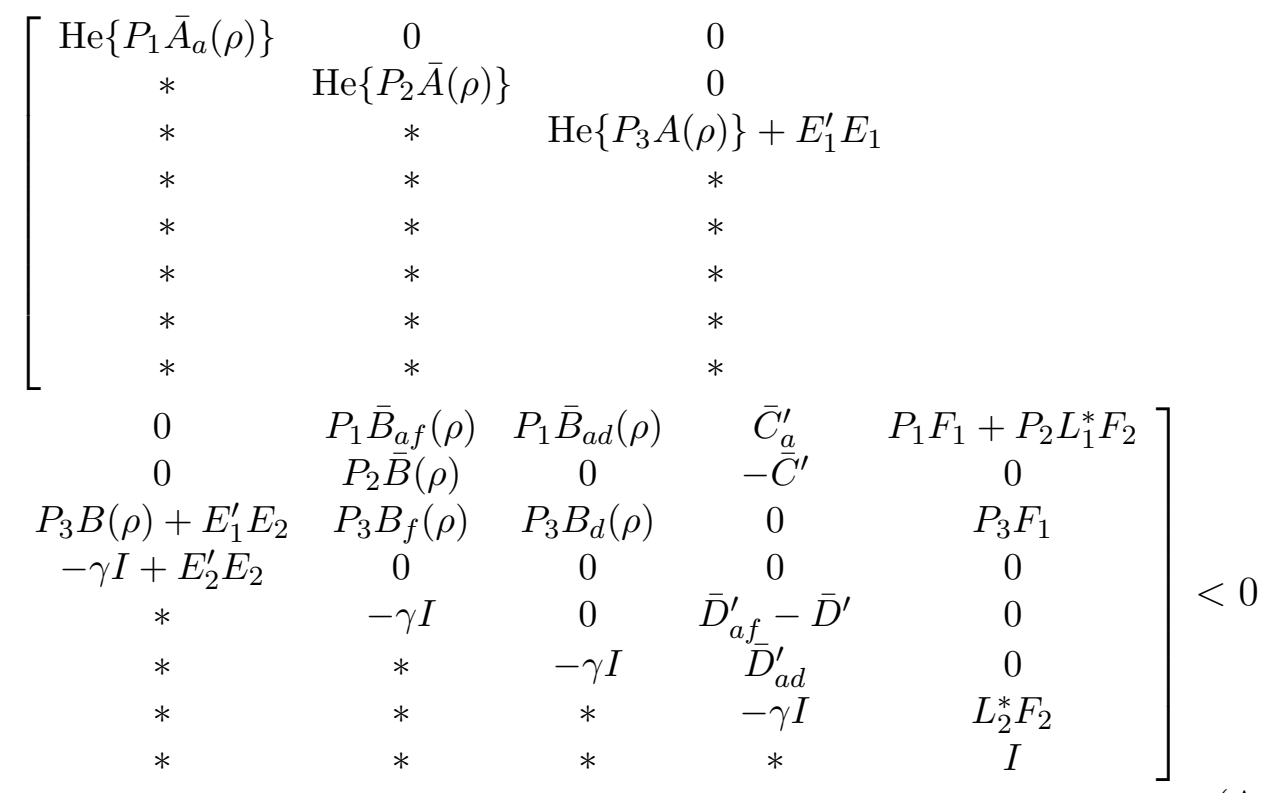

The proof can be completed by substituting Eq.(23) and Eq.(24) into Eq.(A.9) to get Eq.(25). $S^{*}$ is defined as $S^{*}=P_{1} Z_{1}^{*}$. 


\section{References}

Apkarian, P., Gahinet, P., \& Becker, G. (1995). Self-scheduled $\mathscr{H}_{\infty}$ control of linear parameter-varying systems: a design example. Automatica, 31(9), 1251-1261.

Bokor, J., \& Balas, G. J. (2004). Detection filter design for LPV systems - a geometric approach. Automatica, 40(3), 511-518.

Bokor, J., \& Szabo, Z. (2009). Fault detection and isolation in nonlinear systems. Annual Reviews in Control, 33, 113-123.

Chen, J., \& Patton, R. J. (1999). Robust model-based fault diagnosis for dynamic systems. Boston/Dordrecht/London. Kluwer Academic Publishers.

Ding, S. X. (2008). Model-based fault diagnosis techniques: Design schemes, algorithms, and tools. Heidelberg, Berlin: Springer.

Ding, S. X., Jeinsch, T., Frank, P. M., \& Ding, E. (2000). A unified approach to the optimization of fault detection systems. Journal of Adaptive Control and Signal Processing, 17(4), 725-745.

Edwards, C., Lombaerts, T., \& Smaili, H. (2010). Fault tolerant flight control: A benchmark challenge. Springer.

Fernandez, V., De Zaiacomo, G., Mafficini, A., \& Penin, L. F. (2010). The IXV GNC Functional Engineering Simulator. In 11th International Workshop on Simulation 83 EGSE facilities for Space Programmes. ESA-ESTEC.

Fernandez, V., \& Ramon, J. M. (2011). FES software users manual, ADDSAFE Technical Note D1.2.1. Technical Report DEIMOS.

Frisk, E., \& Nielsen, L. (2006). Robust residual generation for diagnosis including a reference model for residual behavior. Automatica, 42(3), 437-445.

Gertler, J. (1998). Fault detection and diagnosis in engineering systems. Marcel Dekker, Inc., New York.

Goupil, P. (2010a). FDD problem definition. Technical Report ADDSAFE Technical Note D.1.1.2.

Goupil, P. (2010b). Oscillatory failure case detection in the A380 electrical flight control system by analytical redundancy. Control Engineering Practice, 18(9), 1110-1119. 
Goupil, P., \& Marcos, A. (2011). Advanced diagnosis for sustainable flight guidance and control: The European ADDSAFE project. In SAE technical paper.

Goupil, P., \& Marcos, A. (2012). Industrial benchmarking and evaluation of ADDSAFE FDD design. In IFAC SAFEPROCESS'12. Mexico City.

Goupil, P., \& Puyou, G. (2011). A high fidelity AIRBUS benchmark for system fault detection and isolation and flight control law clearance. In 4th European Conference for Aerospace Sciences. Saint Petersburg, Russia.

Grenaille, S., Henry, D., \& Zolghadri, A. (2008). A method for designing fault diagnosis filters for LPV polytopic systems. J. Control Sci. Eng., 2008, 1:1$1: 11$.

Hecker, S. (2010). Nominal and faulty LFT/LPV models. Technical Report ADDSAFE Technical Note D.1.3.2-3.

Henry, D. (2008). Fault diagnosis of the MICROSCOPE satellite actuators using $\mathscr{H}_{-} / \mathscr{H}_{\infty}$ filters. AIAA Journal of Guidance, Control and Dynamics, 31(3), 699-711.

Henry, D. (2012). Structured fault detection filters for LPV systems modeled in an LFT manner. International Journal of Adaptive Control and Signal Processing, 26, 190-207.

Henry, D., \& Zolghadri, A. (2005). Design and analysis of robust residual generations for systems under feedback control. Automatica, 41(2), 251-264.

Hou, M., \& Patton, R. J. (1996). An LMI approach to $\mathscr{H}_{-} / \mathscr{H}_{\infty}$ fault detection observers. In Proceedings of the UKACC (pp. 305-310).

Isermann, R. (1997). Trends in the application of model-based fault detection and diagnosis of techinical process. Control Engineering Practice, 5(5), 709-719.

Isermann, R. (2005). Model-based fault-detection and diagnosis status and applications. Annual Reviews in Control, 29(1), 71-85.

Jaimoukha, I. M., Li, Z., \& Papakos, V. (2006). A matrix factorization solution to the $\mathscr{H}_{-} / \mathscr{H}_{\infty}$ fault detection problem. Automatica, 42, 1907-1912.

Li, Z., Mazars, E., Zhang, Z., \& Jaimoukha, I. M. (2012). State-space solution to the $\mathscr{H}_{-} / \mathscr{H}_{\infty}$ fault-detection problem. International Journal of Robust and Nonlinear Control, 22, 282-299. 
Liu, J., Wang, J. L., \& Yang, G. H. (2005). An LMI approach to minimum sensitivity analysis with application to fault detection. Automatica, 41(11), 1995-2004.

Marcos, A., \& Balas, G. J. (2004). Development of linear-parameter-varying models for aircraft. Journal of Cuidance, Control, and Dynamics, 27, 218-228.

Patton, R. J., Frank, P. M., \& Clark, R. N. (2000). Issues in fault diagnosis for dynamic systems. Springer.

Pfifer, H., \& Hecker, S. (2011). Generation of optimal linear parametric models for lft-based robust stability analysis and control design. IEEE Transactions on Control Systems Technology, 19.

Pulecchi, T., \& Marcos, A. (2011). Open loop aircraft LFT models. Technical Report ADDSAFE Technical Note D.1.3.2-1.

Wang, H., \& Yang, G. H. (2008). A finite frequency domain approach to fault detection for linear discrete-time systems. International Journal of Control, $87(7), 1162-1171$.

Wang, J. L., Yang, G. H., \& Liu, J. (2007). An LMI approach to $\mathscr{H}_{-}$index and mixed $\mathscr{H}_{-} / \mathscr{H}_{\infty}$ fault detection observer design. Automatica, 43(9), 1656-1665.

Wei, X., \& Verhaegen, M. (2011). LMI solutions to the mixed $\mathscr{H}_{-} / \mathscr{H}_{\infty}$ fault detection observer design for linear parameter-varying systems. International Journal of Adaptive Control and Signal Processing, 25(2), 114-136.

Zhong, M., Ding, S. X., Lam, J., \& Wang, H. (2003). An LMI approach to design robust fault detection filter for uncertain LTI systems. Automatica, 39(3), 543550.

Zolghadri, A. (2012). Advanced model-based FDIR techniques for aerospace systems: Today challenges and opportunities. Progress in Aerospace Sciences, 53, $18-29$. 

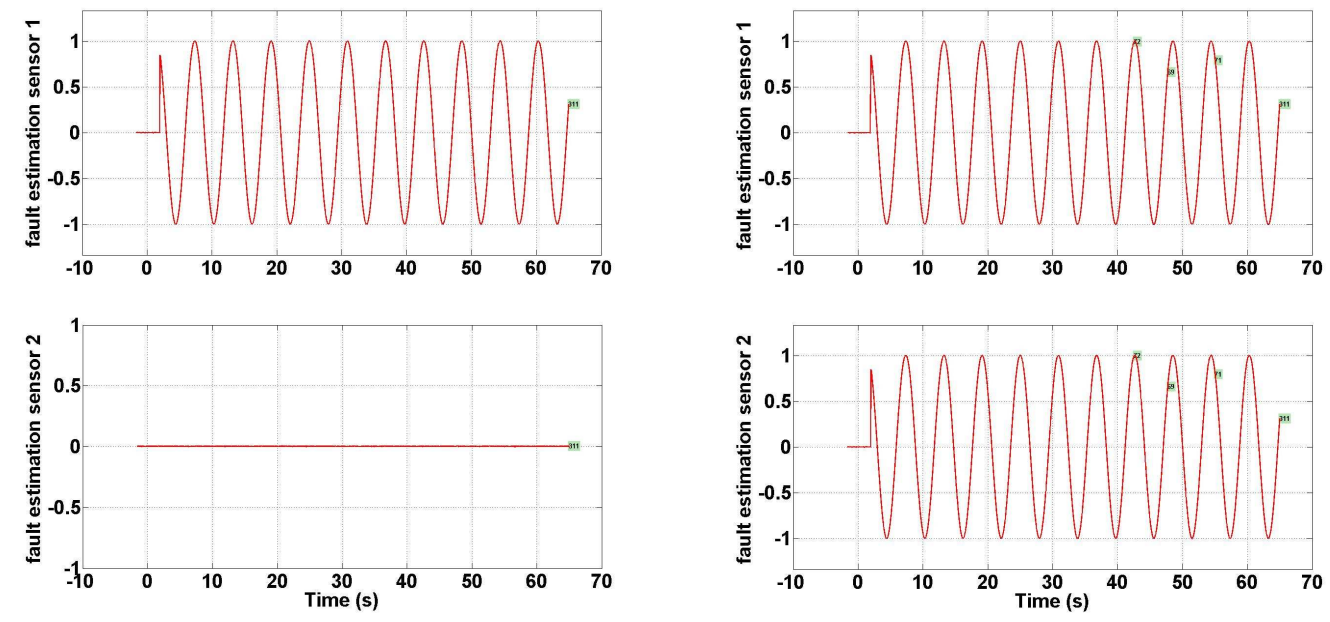

Oscillation A

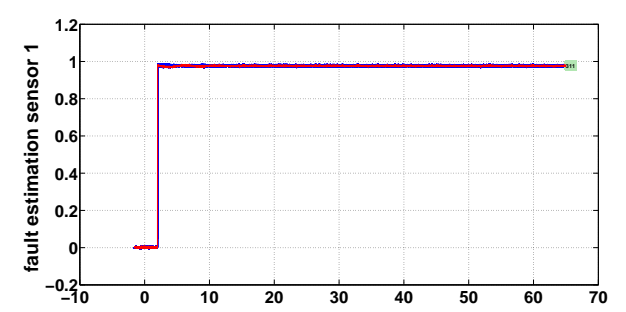

Oscillation B
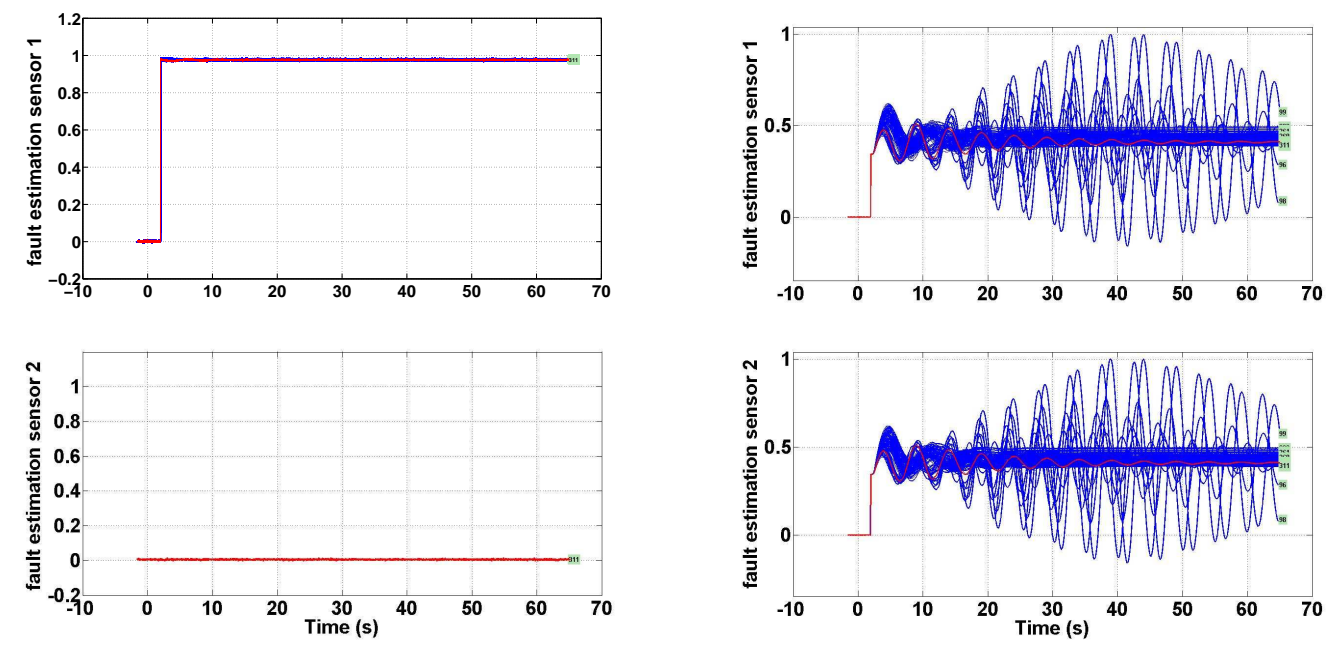

Jamming A

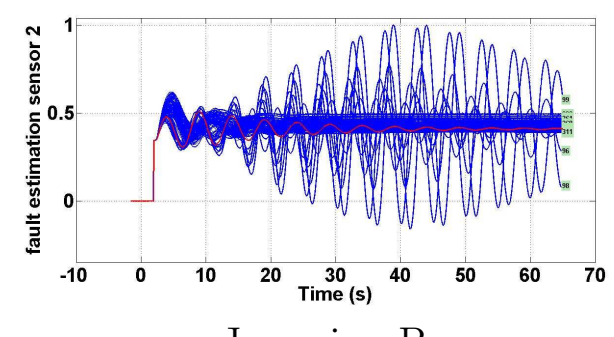

Figure A.3: Fault estimation results of oscillation and jamming fault in Case A and Case B 

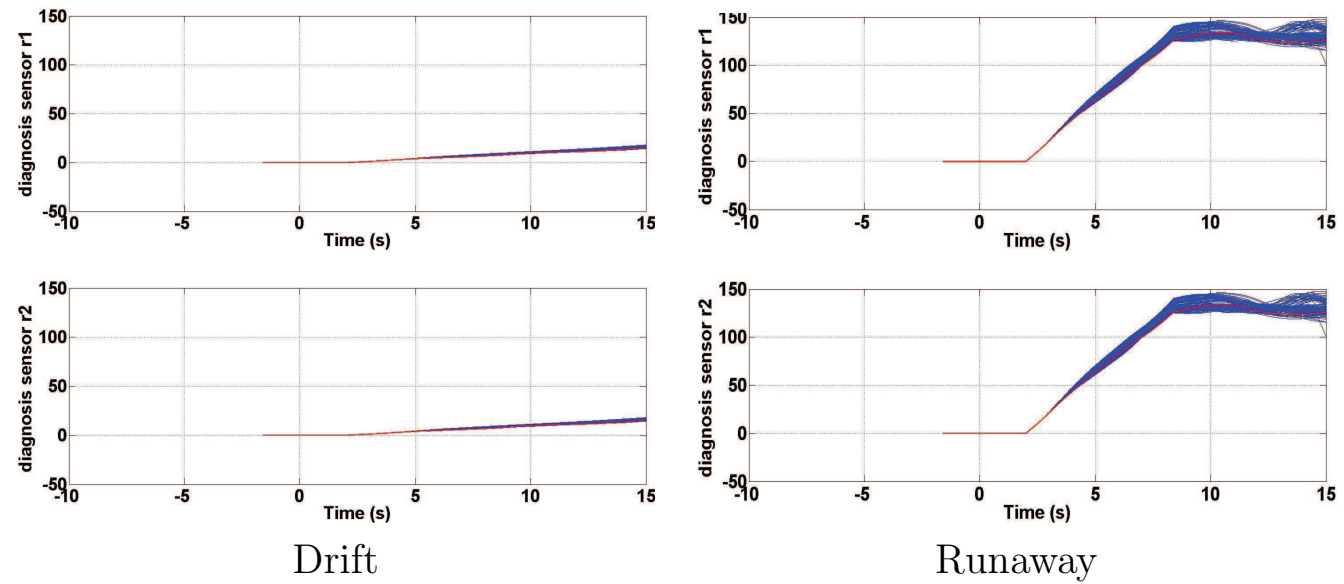

Figure A.4: Fault estimation results of drift and runaway faults in Case B 

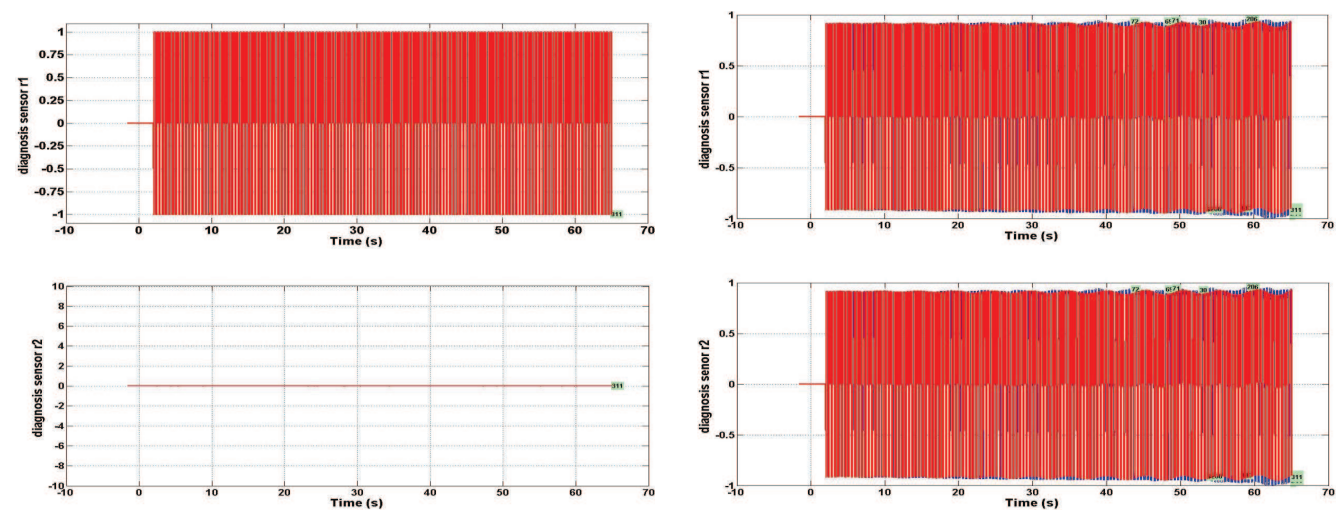

NRZ A

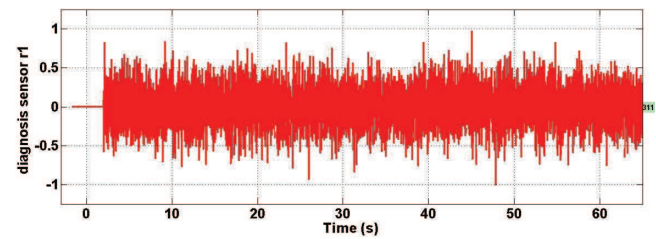

NRZ B
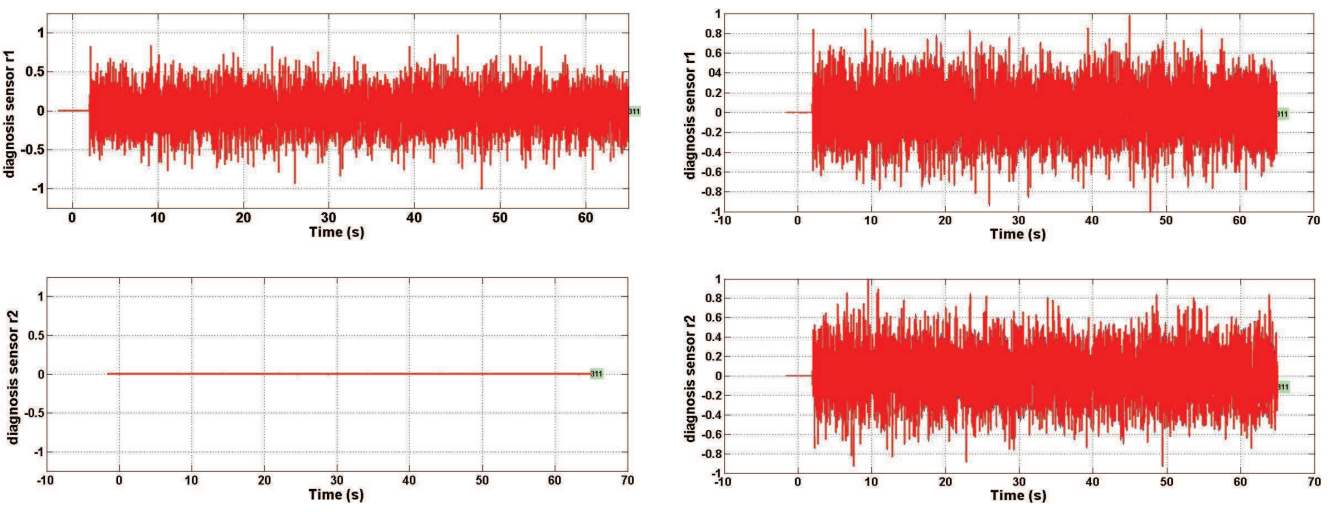

Noise A

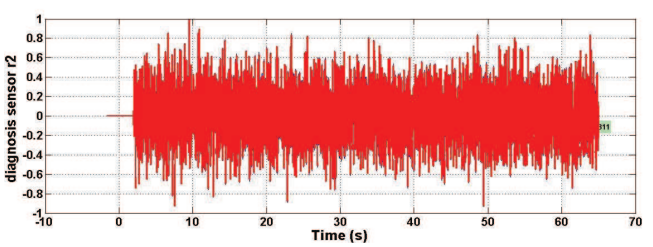

Noise B

Figure A.5: Fault estimation results of NRZ and Noise faults in Case A and Case B 